\title{
EFFECT OF DENTAL BLEACHING DURING ORTHODONTIC TREATMENT ON SURFACE ROUGHNESS OF AESTHETIC BRACKETS: AN ESEM STUDY
}

\author{
Ashraf I. Ali *, Marwa A. Tawfik ${ }^{* *}$ and Enas T. Enan ${ }^{* * *}$
}

\begin{abstract}
Introduction: Fixed orthodontic appliances usually provide a shelter for plaque and stain deposition on teeth, stimulating patients to seek teeth bleaching before finishing their orthodontic treatment course. The influence of the whitening agents on the bonded brackets has not been investigated yet.
\end{abstract}

Aim of the study: The current study was conducted to investigate the effect of bleaching techniques on surface roughness of three different types of esthetic orthodontic brackets.

Materials and methods: Forty five healthy premolars were selected and classified into 3 groups according to the technique of bleaching. Group A was the control un-bleached group, while groups B and C were either subjected to "in-office" or "at-home" bleaching, respectively. For each group, 3 types of brackets were tested; Transcend ${ }^{\circledR}$ (polycrystalline ceramic), Inspire Ice $^{\circledR}$ (Monocrystalline ceramic) and Spirit ${ }^{\circledR}$ (Resin bracket). Brackets were bonded and bleached according to manufacturers' instructions and surface roughness was evaluated using environmental scanning electron microscope (ESEM). For statistical analysis, ANOVA and LSD tests were used.

Results: Brackets subjected to at-home bleaching showed lower Ra values than those subjected to in-office bleaching. Ice ${ }^{\circledR}$ brackets showed the lowest Ra values, in comparison to the other tested types of brackets.

Conclusion: At- home bleaching had lower roughening effect on orthodontic brackets, as compared to in-office technique. Ice brackets were most resistant to the roughening effect of the investigated bleaching agents.

KEYWORDS: Bleaching, Brackets, Surface roughnes.

* Lecturer, Department of Orthodontics, Faculty of Dentistry, Mansoura University, Mansoura, Egypt.

** Lecturer, Department of operative Dentistry, Faculty of Dentistry, Mansoura University, Mansoura, Egypt.

*** Associate professor, Department of Dental Biomaterials, Faculty of Dentistry, Mansoura University, Mansoura, Egypt. 


\section{INTRODUCTION}

One of the factors for dissatisfaction among orthodontic patients is the enamel discoloration during the treatment period, which may last for years. ${ }^{1,2}$ Teeth staining during fixed orthodontic treatment, may be associated with the high plaque accumulation and stain absorption into teeth surfaces, ${ }^{3}$ or it may be related to discoloration of the luting material used for bracket bonding ${ }^{4}$, or even caused by demineralization of the enamel. ${ }^{5}$ Therefore, the demand for tooth whitening among patients under orthodontic treatment has increased exponentially in recent years. ${ }^{5,6}$

In earlier days, dental professionals used to postpone the whitening procedure to be performed after completion of the orthodontic treatment, as they believed that the presence of brackets would impair diffusion of the whitening agent into tooth surface. ${ }^{7}$ 8 In 1990, Haywood et al., reported that the hydrogen peroxide can diffuse laterally even underneath existing restorations. ${ }^{9}$ Similarly, more recent studies also concluded that the bleaching agent is able to travel laterally by diffusion through the tooth structure even in presence of orthodontic brackets. 10-12 Thus, the new concept of simultaneous teeth bleaching during orthodontic treatment was settled.

The effectiveness of bleaching procedures during orthodontic treatment was previously tested and approved by earlier studies. ${ }^{12,13}$ Yet, there is still some concern regarding the influence of the bleaching agent on surface properties of the bonded brackets. Surface roughness is a property of major concern in any esthetically-sensitive situations, such as orthodontic patients, as it may contribute to more problems such as increased plaque accumulation, staining and discoloration. ${ }^{14}$ Thus, the current study was designed to investigate the effect of two different bleaching techniques, in-office and athome, on the surface roughness of three types of esthetic orthodontic brackets. Our null hypothesis assumed that bleaching methods will not cause statistically significant differences regarding surface roughness of the bonded brackets.

\section{MATERIALS AND METHODS}

\section{Specimens' preparation and grouping}

A total of 45 healthy upper and lower premolars, Obtained from out patients' clinic, extracted for orthodontic treatment, were selected for the current study, stored in a $0.1 \%$ thymol solution after cleaning.

The collected teeth were randomly divided into three groups $(n=15)$, according to the technique of bleaching, as follows:

Group A: was not subjected to bleaching (control).

Group B: was subjected to chemically-activated in-office bleaching using 38\% hydrogen peroxide gel Opalescence ${ }^{\circledR}$ Boost PF (Ultradent, South Jordan, UT, USA).

Group C: was subjected to home-bleaching using $10 \%$ hydrogen peroxide gel, Opalescence ${ }^{\circledR}$ Treswhite Supreme ( Ultradent, South Jordan, UT, USA).

Before application of the bleaching procedures, each of the three main groups was equally divided, according to the type of the tested brackets, into 3 subgroups, as follows:

Subgroup 1: Polycrystalline ceramic bracket, Transcend ( 3M Unitek ${ }^{\circledR}$, Monrovia, California).

Subgroup 2: Monocrystalline ceramic bracket, Inspire Ice (Ormco ${ }^{\circledR}$, Orange, California).

Subgroup 3: Resin bracket, Spirit MB ( Ormco ${ }^{\circledR}$, Orange, California).

\section{Application of Brackets}

To facilitate handling, specimens were embedded into acryl blocks with the buccal surface facing outside. For all tested brackets, the same adhesive (TransBond Plus, 3M Unitek, Landsberg, Germany) and the same luting composite (Transbond XT, 3M Unitek, Landsberg, Germany) were applied.

Teeth were etched using 35\% phosphoric acid gel for 30 seconds, washed and dried. Then the primer was applied to the etched tooth surface. The 
light-cure luting composite was then applied to the bracket base, which was fitted to the tooth with a standardized load of $500 \mathrm{~g}$. Excess material was removed carefully. Light curing was done for 60 seconds (15 seconds in each of the gingival, incisal, distal, and mesial directions) using LED curing unit (Epilar Freelight II, 1000 mW/cm2, 3M ESPE, Seefeld, Germany).

\section{Bleaching procedure}

Bleaching procedures were applied according to manufacturer's instructions.

For in-office bleaching, the bleaching agent was mixed to the activator using the supplied syringe. Then the mixture was applied to group B specimens in a layer of $0.5-1 \mathrm{~mm}$ thickness for 20 minutes after which the bleaching material was removed using suction and gauze only. The process was repeated twice a day for a total of 5 consecutive days. After each daily bleaching cycle, specimens were rinsed with water for 1 minute, dried and then stored in distilled water till the next application.

For home-bleaching, specimens of group $\mathrm{C}$ with the bonded brackets were exposed to the supplied bleaching solution for 60 minutes daily for 14 successive days. A pre-loaded bleaching agent in a tray was used in this study, but to prevent contamination of the acrylic blocks, in which the specimens are embedded, with the bleaching agent, specimens were not placed into the tray. The bleaching solution was transferred from the tray and uniformly speeded on the surface of the specimens, with the help of a cotton applicator, in a layer of 1-2 mm thickness. During the bleaching period, specimens were kept in dark containers, to simulate the intra-oral at-home bleaching conditions.

\section{Evaluation of surface roughness}

Surfaces of the tested brackets were assessed using an environmental scanning electron microscope (ESEM) (QUANTA 200, FEI CO., OR, USA) combined with image analysis software (XT document; X-ray tungsten filament document for microanalysis measurements) to provide both qualitative and quantitative assessments of surface roughness. Specimens were photomicrographed at X1000 magnification, and the images were analyzed quantitatively using the image analysis software. A three-dimensional surface roughness (SR) profile was automatically plotted. At the Z-axis, the peaks or surface elevations were marked, and the height of each peak was automatically computed. Mean SR values ( $\mathrm{Ra}$ ) were calculated for each specimen. $\mathrm{Ra}$ describes the arithmetic mean of all values of the roughness profile $(\mathrm{R})$ over the evaluated length. ${ }^{14}$

\section{Statistical analysis}

Statistical analysis software (SPSS 12.0, SPSS, Chicago, USA) was used to analyze the collected data. Data were tested by one-way analysis of variance (ANOVA) and LSD post hoc tests, at a significance level of $5 \%$.

\section{RESULTS}

Means and standard deviations obtained from the surface roughness test are shown in Table 1 . The highest Ra mean value was recorded for resin brackets after in-office bleaching $(39.80 \mu \mathrm{m})$. While the lowest mean value was recorded for Ice brackets without bleaching $(25.90 \mu \mathrm{m})$. Comparing the effect of each bleaching method on the 3 types of brackets, by one-way ANOVA test, showed significant differences between the 3 subgroups among the control group $(\mathrm{p}=0.02)$, after in-office bleaching $(\mathrm{p}<0.0001)$ and also after at-home bleaching $(\mathrm{p}=0.0006)$. Least significant difference (LSD) test of both the control and at-home groups showed significant differences between resin and ceramic brackets and also between resin and ice brackets, while the difference between ice and ceramic brackets was insignificant. For the in-office bleaching group, LSD test showed a significant difference between each of the three types of brackets.

Comparing the effect of bleaching methods on each type of brackets, ANOVA test showed a significant difference between the three groups (control, in-office and at-home) only in the resin brackets subgroup $(\mathrm{p}=0.03)$, while insignificant 
differences between control, in-office and at-home bleaching groups, were found for both the ceramic $(\mathrm{P}=0.709)$ and ice brackets $(\mathrm{P}=0.152)$.

Further analysis of the resin brackets subgroup using LSD test, showed significant differences between the in-office bleaching group and both the control and at-home groups. On the other hand, the results of home bleaching of the resin brackets showed an insignificant difference from the control group.

In figures 1 through 6, 2-D ESEM micrographs and 3D histograms showed the same results presented in Table 1.

TABLE (1) Surface roughness (Ra) values results in $\mu \mathrm{m}$

\begin{tabular}{|c|c|c|c|c|c|}
\hline \multirow{2}{*}{ Type of bracket } & \multicolumn{3}{|c|}{ Mean \pm SD } & \multirow{2}{*}{ F-value } & \multirow{2}{*}{ P-value } \\
\hline & Control & Office & Home & & \\
\hline Ceramic & $29.11^{\mathrm{B}} \pm 3.79$ & $31.54^{\mathrm{B}} \pm 4.81$ & $30.14^{\mathrm{B}} \pm 2.35$ & 0.35 & 0.709 \\
\hline Ice & $25.90^{\mathrm{B}} \pm 2.85$ & $27.12^{\mathrm{C}} \pm 5.82$ & $26.59^{\mathrm{B}} \pm 4.28$ & 2.02 & 0.152 \\
\hline Resin & $33.14^{\mathrm{A}} \pm 4.35$ & $39.80^{\mathrm{A}} \pm 2.56$ & $34.25^{\mathrm{A}} \pm 3.38$ & 8.4 & 0.03 \\
\hline F-value & 9.5 & 15.4 & 17.9 & & \\
\hline P-value & 0.02 & $<0.0001$ & 0.0006 & & \\
\hline
\end{tabular}

Means with the same superscript letter in the same column are not significantly different at $P \leq 0.05$

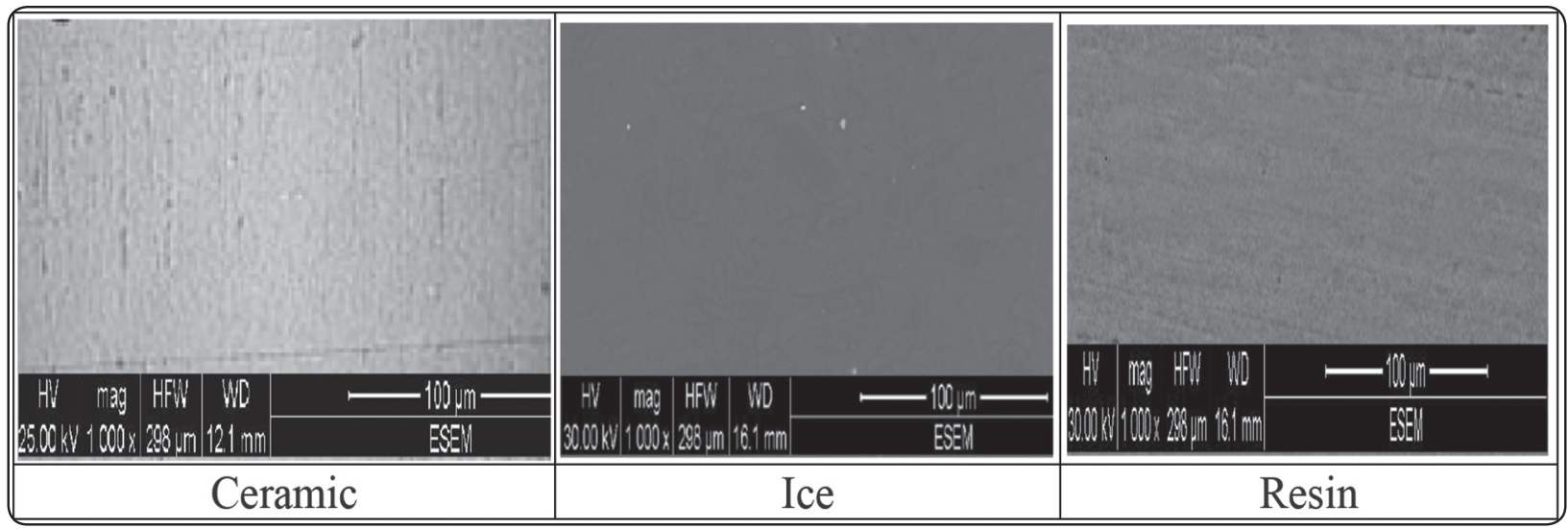

Fig. (1) ESE micrographs of the control group for three types of brackets

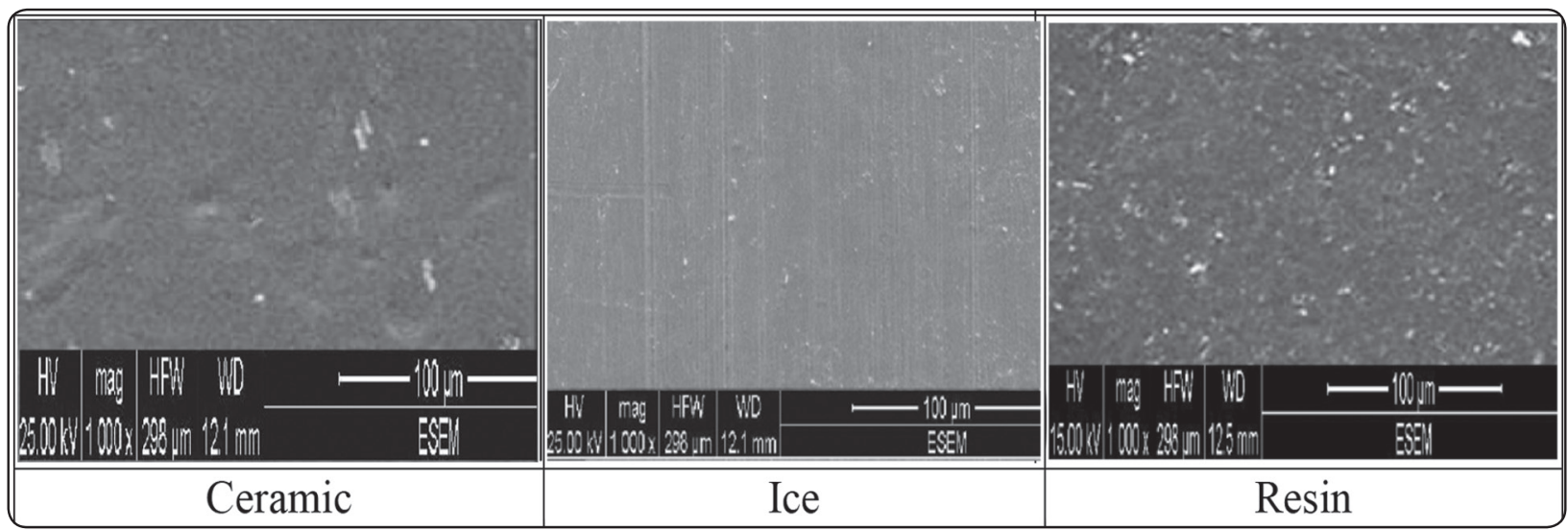

Fig. (2) ESE micr ographs of in-office bleaching group for three types of brackets 


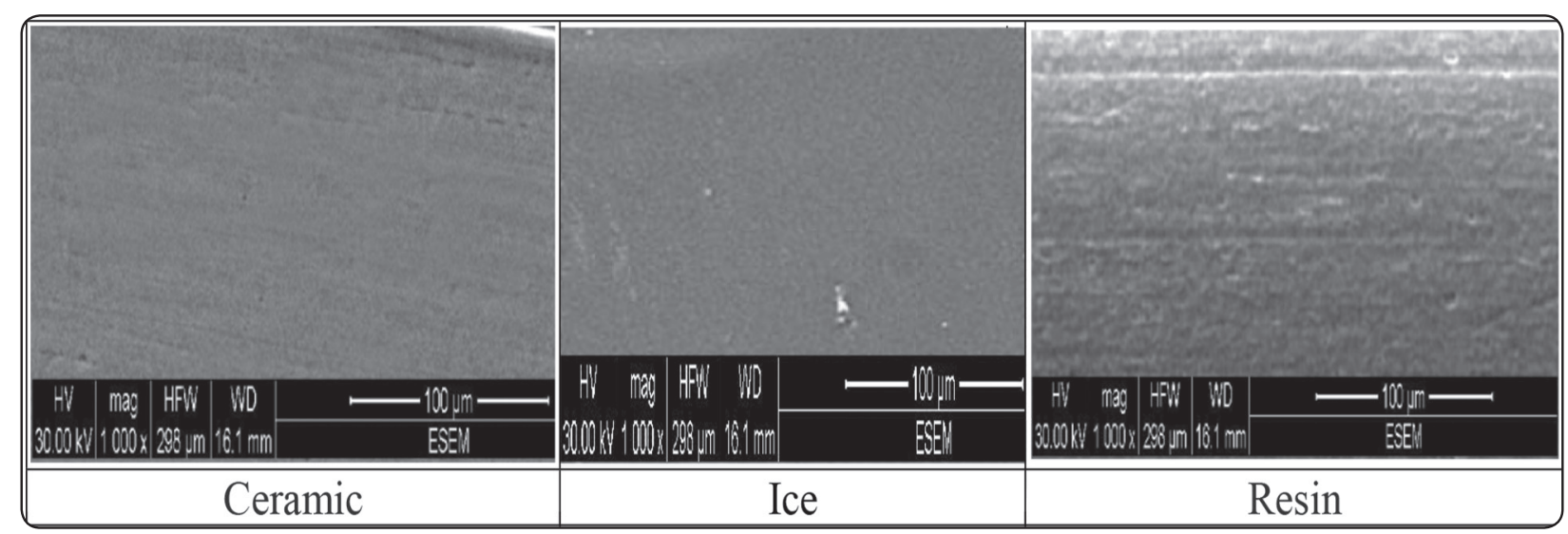

Fig. (3) ESE micrographs of at-home bleaching group for three types of brackets

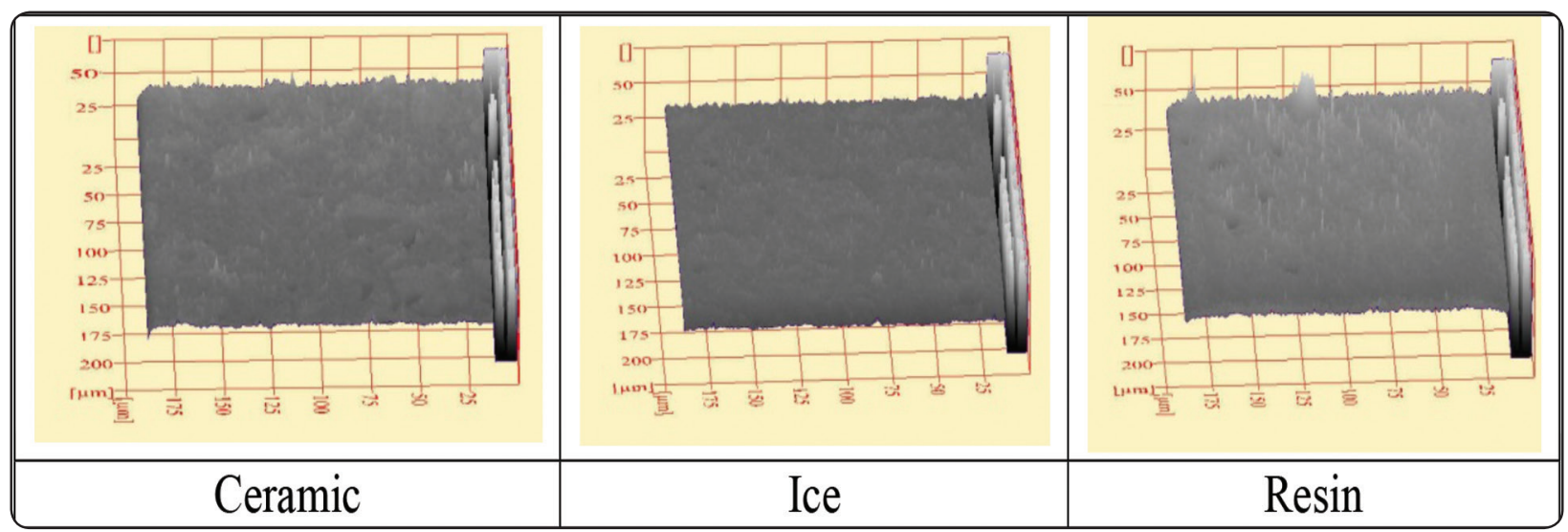

Fig. (4) ESEM histograms of the control group for three types of brackets

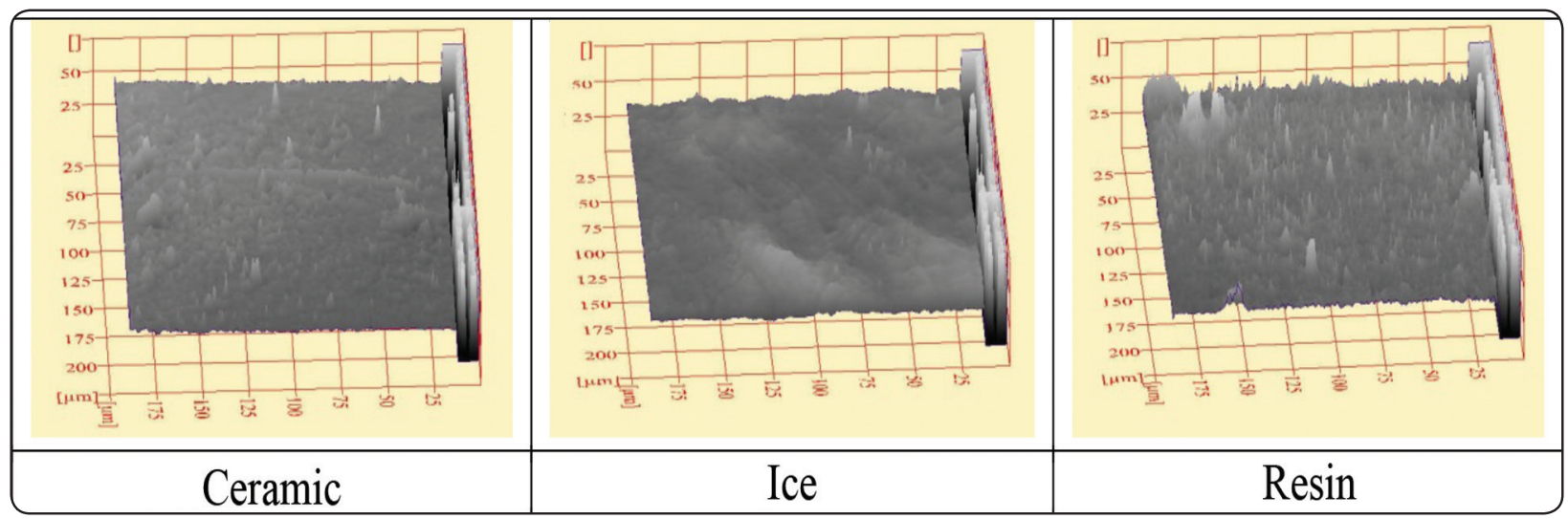

Fig. (5) ESEM histograms of in-Office bleaching group for three types of brackets 


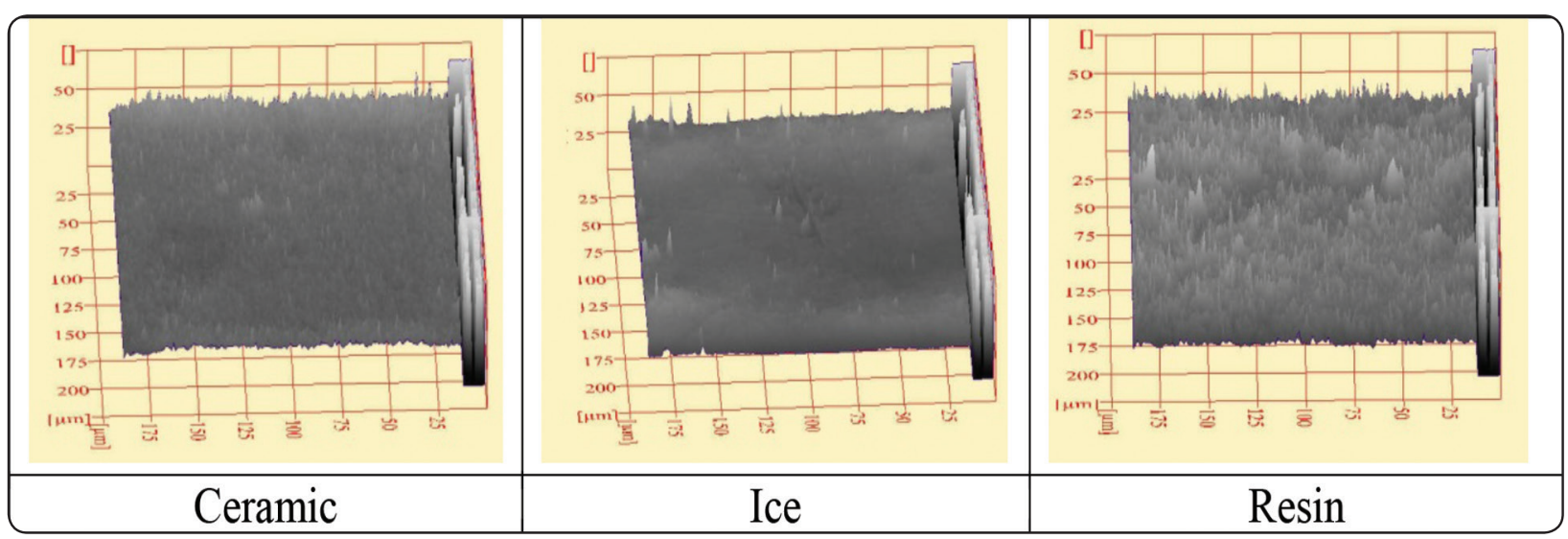

Fig. (6) ESEM histogram of at-home bleaching group for three types of brackets

\section{DISCUSSION}

One of the main motivating factors for patients to seek orthodontic treatment is their desire to gain a beautiful smile with properly-aligned teeth at the end of their treatment course. ${ }^{1}$ Thus, satisfying the esthetic demands of these patients puts a big responsibility on the dental professionals to achieve and maintain their patients' expectations. ${ }^{1-3}$ Unfortunately, fixed orthodontic appliances usually provide a shelter for plaque and stain deposition, which may impulse patients to seek teeth bleaching during their orthodontic treatment course. ${ }^{3,15}$ Previously, dental professionals believed that bleaching during orthodontic treatment is not possible as the bonded brackets may jeopardize diffusion of the bleacher into enamel. 7, 16 More recently, awareness about the ability of bleaching agents to diffuse through dental tissues, even in presence of orthodontic brackets, encouraged many orthodontists to apply dental whitening with fixed orthodontic appliances in place. , $^{3,14}$

Previous studies were conducted to evaluate the efficiency of dental bleaching during orthodontic treatment on de-staining of the treated teeth. ${ }^{9-12}$ However, the influence of teeth whitening on the bonded brackets have not been investigated yet. Thus, the current study was conducted to investigate the effect of bleaching techniques on surface roughness of three different types of esthetic orthodontic brackets. The brackets involved in this study, were selected on the basis of their popularity among orthodontic patients.

Surface roughness property was specifically investigated in the current study because it can have a great influence on the orthodontic appliance. Besides the effect of surface roughening on plaque and stain accumulation on orthodontic brackets, with subsequent discoloration and esthetic impairment, the case may be even worse if the bracket slot is affected. Increased roughness of the bracket slot can change the coefficient of friction (COF) and resistance to sliding (RS) between brackets and arch wires, which subsequently impairs the orthodontic forces transmitted to teeth. ${ }^{17-19}$

In the present study, surface roughness of three types of brackets; Ice, ceramic and resin, were investigated under two different bleaching methods; in-office and ah-home, compared to an unbleached group. Our results showed that the mono-crystalline ceramic (Ice), had and maintained the lowest surface roughness under the applied bleaching conditions. Poly-crystalline ceramic brackets showed intermediate amounts of surface changes between those of Ice and resin ones. These results indicated the high resistance of Ice brackets, to surface changes by different concentrations of 
bleaching agents, because of their most inert nature. Nevertheless, the increased roughness of polycrystalline ceramic on bleaching may be attributed to the reduction of surface silica content..$^{20,21}$

On the other hand, resin brackets showed the highest surface roughness among the three bracket materials, which was further increased especially by in-office bleaching. These results can be attributed to the organic nature of resin brackets which renders them more prone to chemical alteration by the acidic component of bleaching agents. ${ }^{22}$ The present results came in agreement with previous studies, ${ }^{23-25}$ which reported that ceramics had higher ability to maintain their surface stability, even with bleaching, in comparison to resin materials.

Comparing the roughening effect bleaching techniques with each other and with the control (unbleached) group, showed that the highest roughness was caused by the in-office bleaching technique. However, the difference between the three tested groups was significant only when performed on resin brackets. The higher roughening effect of in-office bleaching can be attributed to the higher concentration of HP bleaching agent (38\%) as compared to that of at-home bleaching method $(10 \% \mathrm{HP})$, as this higher concentration is expected to promote faster tooth whitening. ${ }^{26-28}$

Based on the above data, the null hypothesis that assumed that the bleaching methods have no effect on surface roughness of the bonded brackets was rejected. However, our results were not in agreement with Gurbuz et al ${ }^{29}$ and Yu et al ${ }^{30}$ as they found no effect of HP bleaching on any of the materials they investigated. This contrast can be explained in terms of mismatching in the research conditions and methodology.

\section{CONCLUSION AND RECOMMENDATIONS}

Within limitations of the present study, it could be concluded that at- home bleaching technique, when properly applied, had lower effect on surface roughness of orthodontic brackets, as compared to in-office technique. Resin brackets were more prone to roughening by the influence of HP bleaching agent, while mono-crystalline Ice brackets were most resistant to surface changes by the same agent at both of the investigated concentrations. Thus it is recommended for patients with resin brackets whether to delay teeth bleaching until they get their brackets removed, or to use lower concentration at-home bleaching products. Further studies are needed to investigate the effect of other forms and concentrations of teeth bleaching products.

\section{REFERENCES}

1. Karamouzos A, Athanasiou AE, Papadopoulos MA, Kolokithas G. Tooth-color assessment after orthodontic treatment: a prospective clinical trial. Am J Orthod Dentofacial Orthop. 2010; 138: 537-8.

2. Trakyali G, Özdemir FI, Arun T. Enamel colour changes at debonding and after finishing procedures using five different adhesives. Eur J Orthod 2009;31:397-401.

3. Jadad E, Montoya J, Arana G, et al. Spectrophotometric evaluation of color alterations with a new dental bleaching product in patients wearing orthodontic appliances. Am J Orthod Dentofacial Orthop 2011; 140:43-7.

4. Faltemeier A, Rosentrit M, Reicheneder C, Behr M. Discolouration of orthodontic adhesives caused by food dyes and ultraviolet light. Eur J Orthod 2008; 30: 89-93.

5. Knösel M, Attin R, Becker K, Attin T. External bleaching effect on the color and luminosity of inactive white-spot lesions after fixed orthodontic appliances. Angle Orthod. 2007; 77: 646-52.

6. Lia Mondelli RF, Garrido Gabriel TR, Piola Rizzante FA, Magalhães AC, Soares Bombonatti JF, Ishikiriama SK. Do different bleaching protocols affect the enamel microhardness? Eur J Dent. 2015; 9: 25-30.

7. Consolaro A, Consolaro RB, Francischone L. Clarifications, guidelines and questions about the dental bleaching "associated" with orthodontic treatment. Dental Press J Orthod 2013; 18: 4-10.

8. Lunardi N, Correr AB, Rastelli AN, et al. Spectrophotometric evaluation of dental bleaching under orthodontic bracket in enamel and dentin. J Clin Exp Dent 2014; 6: 321-6. 
9. Haywood VB, Leech T, Heymann HO, et al. Nightguard vital bleaching: effects on enamel and surface texture and diffusion. Quintessence Int 1990;21:801-4.

10. Dezotti MS, Souza MH, Jr, Nishiyama CK. Evaluation of $\mathrm{pH}$ variation and cervical dentin permeability in teeth submitted to bleaching treatment. Pesqui Odontol Bras 2002;16:263-8.

11. Palo RM, Palo I, Bonetti-Filho MC, et al. Quantification of peroxide ion passage in dentin, enamel, and cementum after internal bleaching with hydrogen peroxide. Oper Dent 2012;37:660-4.

12. Mauricio G, Hélio D, Alexandre M, Ricardo S \& Devito-Moraes AG. In-Office Bleaching During Orthodontic Treatment. J Esth Rest Dent 2017 ; 29: 83-92.

13. Staley RN, Vargas MA. Bleaching during and after orthodontic treatment. Am J Orthod Dentofacial Orthop. 2004;126:19A.

14. Hafez R, Ahmed D, Yousry M, El-Badrawy W, ElMowafy O. Effect of In-Office Bleaching on Color and Surface Roughness of Composite Restoratives Effect of In-Office Bleaching on Color and Surface Roughness of Composite Restoratives. Eur J Dent 2010;4:118-127.

15. Çörekçi B, Irgın C, Malkoc C, Özturk B. Effects of staining solutions on the discoloration of orthodontic adhesives: An in-vitro study. Am J Orthod Dentofacial Orthop. 2010;138:741-6.

16. Hintz J K, Bradley T G, Eliades T. Enamel colour changes following whitening with 10 per cent carbamide peroxide: a comparison of orthodontically-bonded/debonded and untreated teeth. Eur J Orthod. 2001;23:411-5.

17. Clocheret K, Willems G, Carels C, Celis J-P. Dynamic frictional behavior of orthodontic archwires and brackets. Eur J Orthod 2004; 26:163-170.

18. Marques IS, Araujo AM, Gurgel JA, Normando D. Debris, roughness and friction of stainless steel archwires following clinical use. Angle Orthod 2010; 80:521-27.

19. Choi S, Park KH, Cheong Y, Kim HK, Park YG, Park HK. Changes in ultrastructure and properties of bracket slots after orthodontic treatment with bicuspid extraction. Scanning 2011; 33:25-32.
20. Türker SB, Biskin T. The effect of bleaching agents on the microhardness of dental aesthetic restorative materials. J Oral Rehabil 2002; 29: 657-61.

21. Türker SB, Biskin T. Effect of three bleaching agents on the surface properties of three different esthetic restorative materials. J Prosthet Dent 2003; 89: 466-73.

22. Hannig C, Duong S, Becker K, Brunner E, Kahler E, Attin T. Effect of bleaching on subsurface micro-hardness of composite and a polyacid modified composite. Dent Mat 2007; 23: 198-203.

23. Yu H, Li Q, Cheng H, Wang Y. The effects of temperature and bleaching gels on the properties of tooth-colored restorative materials. J Prosth Dent 2011;105:100-7

24. Attin T, Hannig C, Wiegand A, Attin R. Effect of bleaching on restorative materials and restorations - a systematic review. Dent Mat 2004;20:852-61.

25. Ourique SA, Arrais CA, Cassoni A, Ota-Tsuzuki C, Rodrigues JA. Effects of different concentrations of carbamide peroxide and bleaching periods on the roughness of dental ceramics. Braz Oral Res 2011;25:453-8.

26. Sulieman M; An overview of bleaching techniques. 2. Night guard vital bleaching and non-vital bleaching. Dental Update 2005: 32. 39-46.

27. PFL Dawson, MO Sharif, AB Smith, and PA Brunton. A Clinical Study Comparing the Efficacy and Sensitivity of Home vs combined Whitening. Oper Dent 2011, 5, 460-466.

28. Basting RT, Amaral FLB, Franca FMG and Flório FM. Clinical Comparative Study of the Effectiveness of and Tooth Sensitivity to $10 \%$ and $20 \%$ Carbamide Peroxide Home-use and 35\% and 38\% Hydrogen Peroxide In-office Bleaching Materials Containing Desensitizing Agents. Oper Dent 2012, 37-5, 464-473.

29. Gurbuz A, Ozkan P, Yilmaz K, Yilmaz B, Durkan R. Effect of at-home whitening strips on the surface roughness and color of a composite and an ormocer restorative material. J Prosth 2013;22:69-73.

30. Yu H, Li Q, Wang Y, Cheng H. Effects of temperature and in-office bleaching agents on surface and subsurface properties of aesthetic restorative materials. J dent 2013; 41: 1290-1296. 